\title{
CORRESPONDENCE
}

\section{Ubiquitination of SARS-CoV-2 ORF7a promotes antagonism of interferon response}

\author{
Zengguo $\mathrm{Cao}^{1,2}$, Hongjie Xia ${ }^{2}$, Ricardo Rajsbaum ${ }^{3,4}$, Xianzhu Xia ${ }^{1}$, Hualei Wang ${ }^{1}$ and Pei-Yong Shi $\mathbb{D}^{2,4,5,6}$ \\ Cellular \& Molecular Immunology (2021) 18:746-748; https://doi.org/10.1038/s41423-020-00603-6
}

Understanding interactions between the host and SARS-CoV-2 is essential for developing effective vaccines and therapeutics. Here, we report that SARS-CoV-2 usurps the host ubiquitin system to polyubiquitinate accessory protein ORF7a at Lys119. The ORF7a polyubiquitination is primarily formed by K63-linked ubiquitin chains. Such ubiquitination enhances ORF7a to inhibit type-I interferon (IFN-I) signaling via STAT2 phosphorylation.

Protein ubiquitination is a posttranslational modification that regulates many aspects of eukaryotic biology, including viral infection. ${ }^{1}$ This modification plays a key role in modulating the immune response, ${ }^{2}$ and viruses have evolved to evade immune responses by subverting the host ubiquitin system. ${ }^{3,4}$ To date, little is known about whether SARS-CoV-2 utilizes the ubiquitin system to antagonize the innate antiviral response. Coronavirus accessory proteins are well documented as perturbing cellular processes, ${ }^{5}$ among which SARS-CoV-2 ORF7a was recently shown to inhibit IFN-I signaling. ${ }^{6}$ We hypothesized that SARS-CoV-2 hijacks the host ubiquitin system to enhance ORF7a's ability to antagonize IFN-I responses.

To test this hypothesis, we examined whether SARS-CoV-2 ORF7a can be ubiquitinated. HEK293T cells were cotransfected with two plasmids expressing Flag-tagged ORF7a (Flag-ORF7a) and HA-tagged ubiquitin (HA-Ub), and the ORF7a protein was immunoprecipitated and probed for ubiquitin using anti-Flag and -HA antibodies, respectively. Based on the molecular masses of Flag-ORF7a (16 kDa) and HA-Ub ( 8.5 kDa), the ORF7a protein appeared to be polyubiquitinated (Fig. 1a). To demonstrate that ORF7a can also be ubiquitinated by endogenous ubiquitin, we expressed Flag-ORF7a alone, immunoprecipitated it with an anti-Flag antibody, and probed for ubiquitinconjugated ORF7a. Western blot analysis showed that ORF7a was robustly ubiquitinated by endogenous cellular ubiquitin (Fig. S1). Collectively, the results indicate that SARS-CoV-2 ORF7a is ubiquitinated.

SARS-CoV-2 ORF7a has seven Lys residues that may serve as ubiquitination sites. To identify which Lys was ubiquitinated, we mutated the individual Lys residues to Ala and assessed the mutant ORF7a for ubiquitination. Only mutant K119A ORF7a showed substantially reduced ubiquitination (Fig. 1b), suggesting that ubiquitination occurs at residue K119.

Ubiquitin has seven Lys residues that can serve as polyubiquitination points, among which $\mathrm{K} 48$ and $\mathrm{K} 63$ are the most common residues for ubiquitin chain formation. To determine the ubiquitin linkage type, we ectopically expressed ORF7a in the presence of different ubiquitin K-to-R mutants, followed by coimmunoprecipitation analysis (Fig. 1c). Ubiquitination of ORF7a was substantially reduced in the presence of Ub-K63R but not Ub-K48R. Consistent with this result, ORF7a ubiquitination was significantly reduced in the presence of Ub-K48-only (all Lys residues were mutated to Arg except for K48). These results suggest that ORF7a is mostly ubiquitinated with K63-linked polyubiquitin chains. As Ub-K63-only slightly reduced ORF7a ubiquitination, other polyubiquitin types may also be present.

As ORF7a is reported to inhibit IFN-I signaling, ${ }^{6}$ we asked whether ubiquitination is required for this activity. Using an ISREpromoter-driven luciferase assay, ${ }^{6}$ we compared inhibitory activities between WT and ubiquitination-defective K119A ORF7a (Fig. 1d). Upon IFN-a treatment, both proteins inhibited activation of the ISRE promoter in a dose-dependent manner. The K119A ORF7a was significantly less potent at inhibiting IFN-I signaling than WT ORF7a. Consistently, the mRNA levels of IFNstimulated genes (ISGs), including ISG56, IFITM1, and OAS1 (Fig. 1e-g), were higher in cells expressing mutant ORF7a than in those expressing WT ORF7a. These results suggest that ubiquitination at K119 is important for optimal antagonism of IFN-I signaling by ORF7a.

We next sought to determine which step of the IFN-I signaling pathway is inhibited by ORF7a. Upon IFN-a stimulation, WT ORF7a suppressed STAT2 but not STAT1 phosphorylation in a dosedependent manner (Fig. 1h). In contrast, mutant K119A ORF7a did not significantly affect STAT2 phosphorylation, suggesting that ubiquitination is important for optimal suppression of STAT2 phosphorylation. Because phosphorylated STAT1/STAT2 in the ISGF3 complex translocates to the nucleus to activate ISG transcription, ${ }^{7}$ we examined the effect of WT or mutant ORF7a on STAT1 nuclear translocation. WT ORF7a, but not mutant K119A, inhibited the nuclear translocation of STAT1 (Fig. 1i). Altogether, the results suggest that ubiquitination is required for ORF7a to inhibit IFN-a signaling by blocking STAT2 phosphorylation.

Collectively, we show for the first time that SARS-CoV-2 ORF7a usurps the host ubiquitin system to promote antagonism of IFNI responses. Among other SARS-CoV-2 proteins with IFN-I antagonism, ${ }^{6}$ nsp13, ORF3a, and ORF7a also seem to be

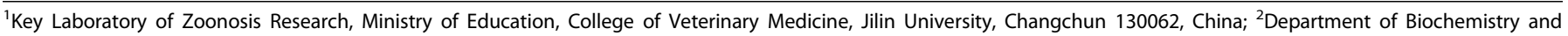

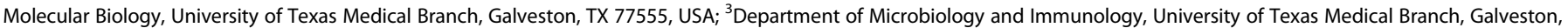

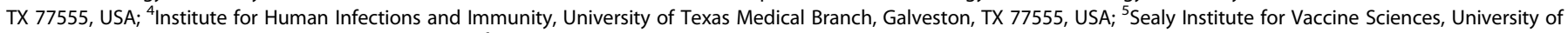

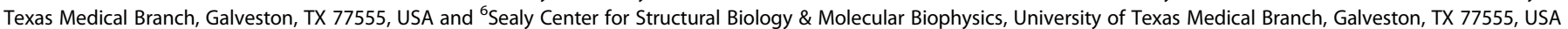
Correspondence: Hualei Wang (wanghualei@jlu.edu.cn) or Pei-Yong Shi (peshi@UTMB.edu)

These authors contributed equally: Zengguo Cao, Hongjie Xia
}

Received: 10 November 2020 Accepted: 20 November 2020

Published online: 20 January 2021 


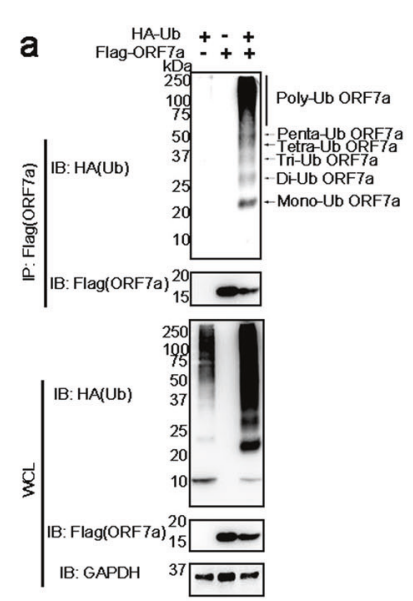

d

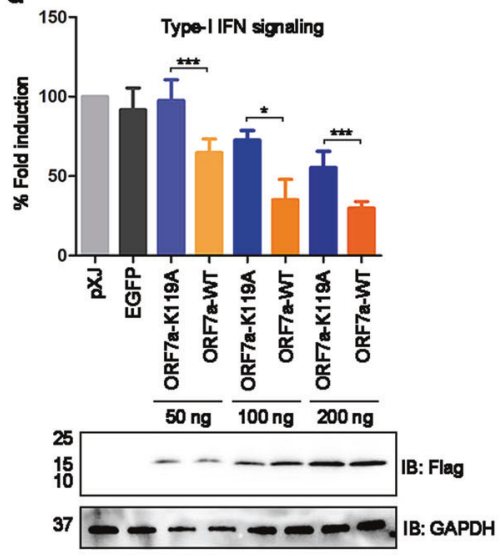

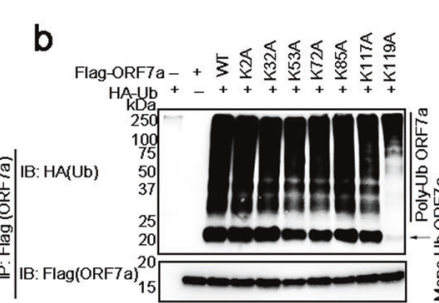
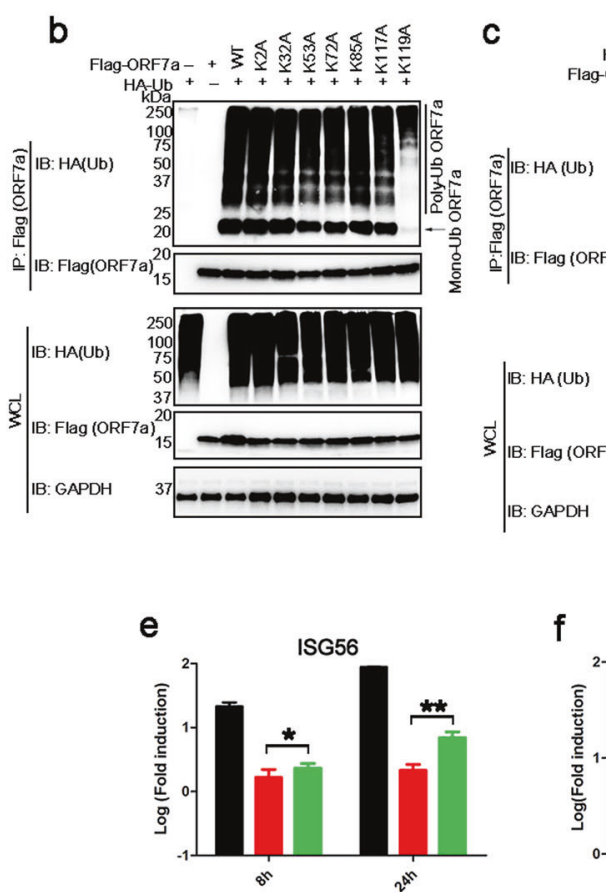

f
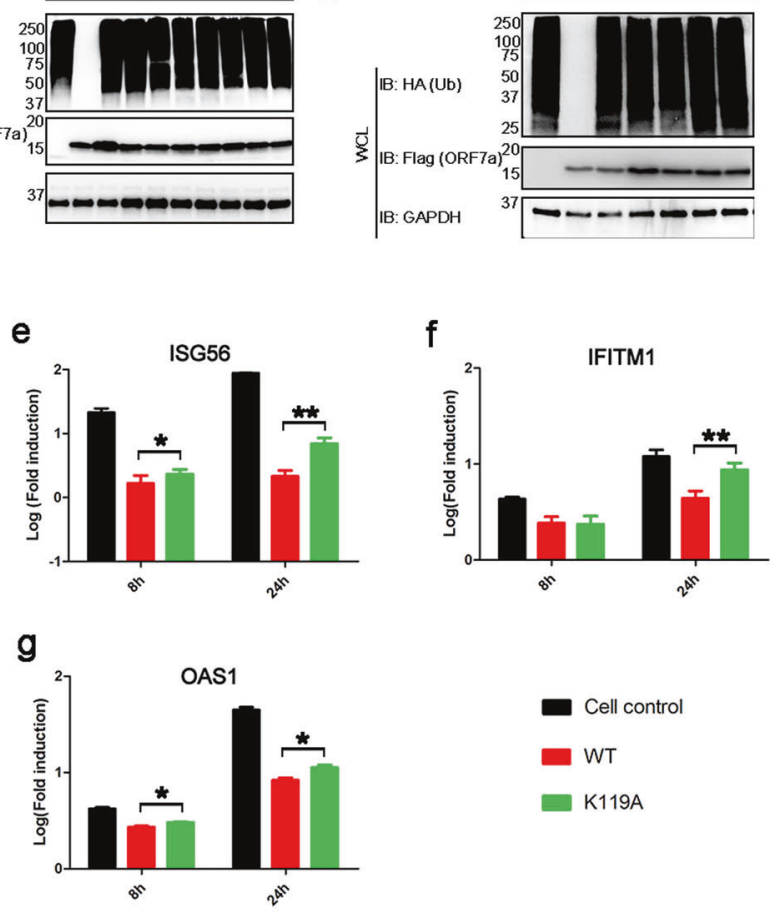

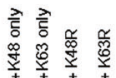

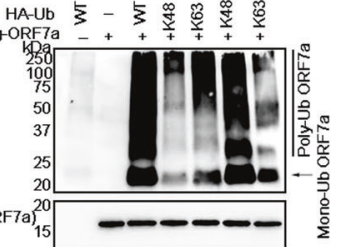

h

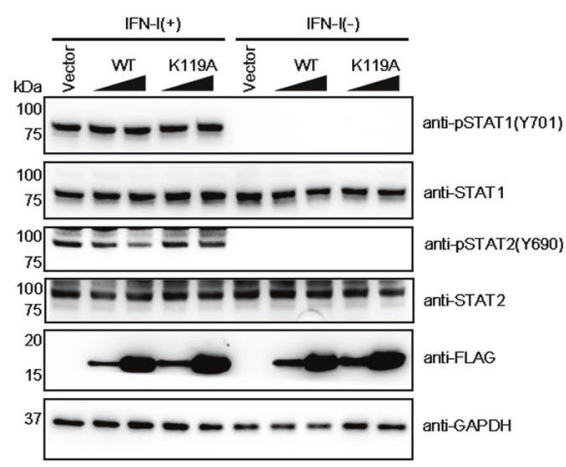

i

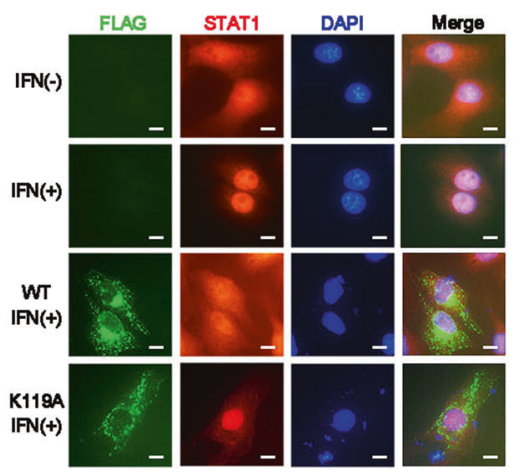

Fig. 1 a HEK293T cells were cotransfected with plasmids expressing Flag-tagged ORF7a and HA-tagged ubiquitin (500-ng DNA each). At 30 $\mathrm{h}$ posttransfection (hpt), cells were lysed, denatured at $70^{\circ} \mathrm{C}$ for $10 \mathrm{~min}$, immunoprecipitated (IP) with an anti-Flag antibody, and probed with an anti-HA antibody by Western blotting. b HEK293T cells cotransfected with plasmids expressing Flag-tagged wild-type (WT) or mutant ORF7a and HA-tagged ubiquitin were analyzed for ORF7a ubiquitination at $30 \mathrm{hpt}$ c HEK293T cells cotransfected with plasmids expressing ORF7a and WT or mutant ubiquitin were examined for ORF7a ubiquitination at $30 \mathrm{hpt}$. d HEK293T cells were cotransfected with an ISRE-promoterdriven firefly luciferase reporter plasmid ( $250 \mathrm{ng}$ ), Renilla luciferase control plasmid (20 ng), and WT or K119A ORF7a-expressing plasmid. At 16 hpt, the cells were treated with human IFN- $\alpha(1000$ units $/ \mathrm{ml})$. After $8 \mathrm{~h}$ of treatment, the cells were assayed for dual-luciferase activities (Promega). The data were analyzed by normalizing firefly luciferase to Renilla luciferase activities and then normalizing to non-IFN- $\alpha$-treated samples to obtain fold induction. The value of the empty vector control was set to $100 \%$-fold induction. Error bars represent mean \pm S.D. e-g HEK293T cells were transfected with a plasmid expressing WT or K119A ORF7a. Untransfected cells were included as controls. At 24 hpt, the cells were treated with human IFN- $\alpha$ (1000 units/ml). At the indicated times after IFN- $\alpha$ treatment, intracellular ISG56, IFITM1, and OAS1 mRNA levels were measured by real-time RT-PCR. The data were first normalized to cellular GAPDH mRNA and then to non-IFN- $\alpha$-treated samples to obtain fold induction. Data are shown as the mean \pm S.D. from three independent experiments. $\mathbf{h}$ HEK293T cells were transfected with the indicated protein-expressing plasmids. At $24 \mathrm{hpt}$, the cells were treated with IFN- $\alpha$ (1000 units/ml) for 30 min and analyzed for STAT1/STAT2 phosphorylation by Western blotting. i Vero cells were transfected with WT or K119A ORF7a-expressing plasmid. At 24 hpt, the cells were treated with IFN- $\alpha$ (1000 units/ml) for $30 \mathrm{~min}$, fixed with $4 \%$ paraformaldehyde, permeabilized with $1 \%$ Triton X-100, and probed with anti-Flag and anti-STAT1 antibodies as primary antibodies and anti-Alexa Fluor 488 and anti-Alexa Fluor 568 as secondary antibodies. Scale bar, 10 mm. Statistical significance was determined by Student's two-sided $t$-test, ${ }^{*} p<0.05,{ }^{* *} p<0.01,{ }^{* * *} p<0.001$ 
ubiquitinated (Fig. S2). Future studies are needed to characterize the molecular details and biological functions of this ubiquitination.

\section{ACKNOWLEDGEMENTS}

We thank P.-.Y.S. lab and other members of the lab at the University of Texas Medical Branch for discussing and analyzing data during this study.

\section{ADDITIONAL INFORMATION}

The online version of this article (https://doi.org/10.1038/s41423-020-00603-6) contains supplementary material.

Competing interests: The authors declare no competing interests.

\section{REFERENCES}

1. Swatek, K. N. \& Komander, D. Ubiquitin modifications. Cell Res. 26, 399-422 (2016).

2. Ebner, P., Versteeg, G. A. \& Ikeda, F. Ubiquitin enzymes in the regulation of immune responses. Crit. Rev. Biochem. Mol. Biol. 52, 425-460 (2017).

3. Rajsbaum, R. \& Garcia-Sastre, A. Viral evasion mechanisms of early antiviral responses involving regulation of ubiquitin pathways. Trends Microbiol. 21, 421-429 (2013)

4. Laurent-Rolle, M. et al. The interferon signaling antagonist function of yellow fever virus NS5 protein is activated by type I interferon. Cell Host Microbe 16, 314-327 (2014).

5. Liu, D. X., Fung, T. S., Chong, K. K., Shukla, A. \& Hilgenfeld, R. Accessory proteins of SARS-CoV and other coronaviruses. Antivir. Res. 109, 97-109 (2014).

6. Xia, H. et al. Evasion of Type I Interferon by SARS-CoV-2. Cell Rep. 33, 108234 (2020).

7. Au-Yeung, N., Mandhana, R. \& Horvath, C. M. Transcriptional regulation by STAT1 and STAT2 in the interferon JAK-STAT pathway. JAKSTAT 2, e23931 (2013). 